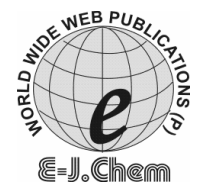

http://www.e-journals.net

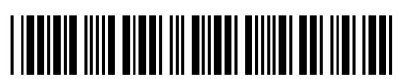

ISSN: 0973-4945; CODEN ECJHAO

E-Journal of Chemistry

2009, 6(1), 1-12

\title{
Review
}

\section{Large Scale Synthesis of Carbon Nanotubes}

\author{
S.KARTHIKEYAN ${ }^{*}$, P. MAHALINGAM ${ }^{\#}$ and M. KARTHIK ${ }^{\S}$
}

*Department of Chemistry, Erode Sengunthar Engineering College, Thudupathi, Erode, India.

\#Department of Chemistry, Sasurie College of Engineering, Vijayamangalam, Erode, India.

${ }^{\S}$ Institute of Environmental Engineering, National Chiao Tung University, Taiwan. skmush@rediffmail.com

Received 11 July 2008; Accepted 1 September 2008

\begin{abstract}
Since the discovery of carbon nanotubes in 1991 by Iijima, they have been of great interest both from the fundamental point of view and for future applications. The most eye catching features of this structure are their electronic, mechanical, optical and chemical characteristics, which opens a way to future applications. These properties can even be measured on single nanotubes. For commercial applications, large quantities of purified nanotubes are needed. In this paper, recent research on preparation of carbon nanotubes with special reference to low temperature synthesis of high purity is reviewed. The reported achievements in this area will open up more knowledge on carbon nanostructured materials in many areas of emerging nanoscale science and nanotechnology.
\end{abstract}

Keywords: Carbon Nanotubes, SWNTs, MWNTs, Arc Discharge method, Laser Ablation method and, Chemical Vapor Deposition method.

\section{Introduction}

Until 1985 it was generally believed that solid elemental carbon occurs in two different crystalline phases: diamond and graphite. Diamond is in thermodynamic equilibrium at very high temperatures and pressures; it occurs, nevertheless, as a metastable phase under atmospheric pressure and at room temperature. In the structure of diamond each carbon atom is tetrahedrally surrounded by four $\mathrm{sp}^{3}$ covalently bonded carbon atoms. The resulting spatial network of carbon is built on a cubic face centred lattice. The structure of graphite consists of graphene layers in which the $\mathrm{sp}^{2}$ bonded carbon atoms form a planar hexagonal honeycomb arrangement. The bonding of carbon atoms in a graphene plane is very strong (covalent bonds), whereas the bonding between two graphene layers is weak (Vander Waals bonds). 
In 1985 an important breakthrough in carbon research was realized by the work of Kroto et al ${ }^{1}$, which resulted in the discovery of large family of all carbon molecules, called 'fullerenes'. They can be crystallized as molecular crystals, which are thus a third form of crystalline elemental carbon. The fullerenes are closed cage carbon molecules with the carbon atoms tiling spherical or nearly spherical surfaces, the best known example being $\mathrm{C}_{60}$ with a truncated icosahedral structure formed by 12 pentagonal rings and 20 hexagonal rings. Carbon nanotubes (CNTs) were discovered by Iijima ${ }^{2}$, who was looking for new carbon structures, in the deposit formed on graphite cathode surfaces during the electric-arc evaporation (or discharge) that is commonly employed to produce fullerene soot. The CNTs, also known as tubular fullerenes, are cylindrical graphene sheets of $\mathrm{sp}^{2}$ bonded carbon atoms. These nanotubes are concentric graphitic cylinders closed at either end due to the presence of five-membered rings. The CNTs can be multiwalled with a central tube of nanometric diameter surrounded by graphitic layers separated by $\sim 0.34 \mathrm{~nm}$. Unlike the multiwalled carbon nanotubes (MWNTs), in single walled carbon nanotubes (SWNTs) there is only the tube and no graphitic layers i.e. SWNTs consist of singular graphene cylindrical walls. Ever since, the discovery of CNTs, several ways of preparing them has been explored. The CNTs have been synthesized by various methods e.g. electric arc discharge, laser evaporation and chemical vapor deposition ${ }^{3,4}$. These methods are very useful and are of widespread importance.

\section{Arc-vaporization}

This method creates CNTs through arc-vaporization of two carbon rods placed end to end, separated by approximately $1 \mathrm{~mm}$, in an enclosure that is usually filled with inert gas at low pressure (Figure 1). A direct current of 50 to 100A, driven by a potential difference of approximately $20 \mathrm{~V}$, creates a high temperature arc discharge between the two electrodes. The arc provides high temperatures which are needed to vaporize carbon atoms into a plasma $\left(>3000^{\circ} \mathrm{C}\right)^{3,5,6}$.

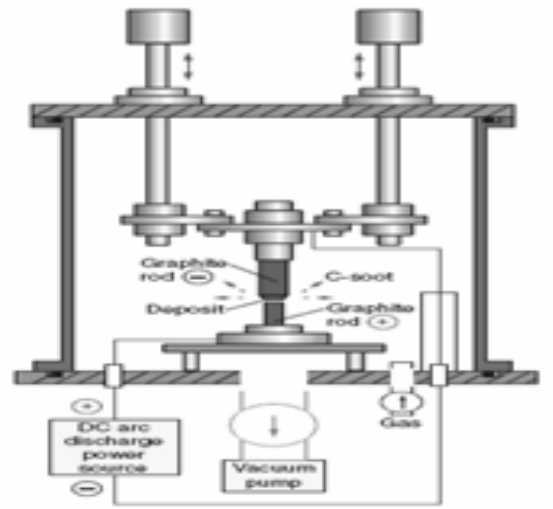

Figure 1. Schematic diagram of arc discharge method.

The discharge vaporizes the surface of one of the carbon electrodes, and forms a small rod-shaped deposit on the other electrode ${ }^{7}$. The arc-discharge method is the one by which CNTs were first produced and recognized. The history of CNTs is closely related to the mass production of fullerenes developed by Kratschmer ${ }^{8}$ in 1990. Soon after, the studies with a dc arc voltage ${ }^{9}$ between two separated graphite rods were carried out. Evaporated anode generates fullerens ${ }^{10}$ in the form of soot in the chamber and a part of the evaporated anode is deposited on the cathode. In that cathode deposit, Iijima found the treasure, CNTs ${ }^{2}$. 
The yield of CNTs depends on the stability of the plasma formed between the electrodes, the current density, inert gas pressure and cooling of electrodes and chamber ${ }^{3,6}$. Among the various inert gases, helium $(\mathrm{He})$ gives the best results, probably due to its high ionization potential $^{11}$. Large-scale synthesis of MWNTs by arc discharge has been achieved ${ }^{3,12}$ in He gas.

When pure graphite rods are used, the anode evaporates to form fullerenes, which are deposited in the form of soot in the chamber ${ }^{10}$. However, a small part of the evaporated anode is deposited on the cathode, which includes CNTs. When a graphite rod containing metal catalyst $(\mathrm{Fe}, \mathrm{Co}$, etc. $)$ is used as the anode with a pure graphite cathode, single-walled carbon nanotubes (SWNTs) $)^{13,14}$ are generated in the form of soot.

The CNTs, made of coaxial graphene sheets and called multiwalled carbon nanotubes (MWNTs), are found not only on the top surface of the cathode deposit ${ }^{15}$ but also deep inside the deposit ${ }^{9}$. Ando, has carried out the arc-discharge evaporation of pure graphite rods in various kinds of ambient gases $(\mathrm{He}, \mathrm{Ar} \text {, and } \mathrm{CH} 4)^{16}$ since $\mathrm{CNTs}$ were first discovered. MWNTs with high crystallinity and few coexisting carbon nanoparticles ${ }^{16}$ were formed with methane. On the contrary, fullerenes cannot be produced in gas including hydrogen atoms ${ }^{17}$. This is the essential difference between CNT and fullerene production. The role of gas including hydrogen atoms in MWNT production is clarified based on analysis of ambient methane gas before and after arc discharge by mass spectroscopy ${ }^{18}$, which revealed that the thermal decomposition of $\mathrm{CH}_{4}$ gas generates hydrogen gas in situ. $2 \mathrm{CH}_{4} \rightarrow \mathrm{C}_{2} \mathrm{H}_{2}+3 \mathrm{H}_{2}$. Therefore, pure graphite rods were arc evaporated in pure hydrogen gas ${ }^{19,20}$. The effectiveness of hydrogen arc discharge in producing MWNTs with high crystallinity was confirmed ${ }^{20}$, and a new morphology of carbon, the 'carbon rose', was also found ${ }^{19}$. A similar effect of ambient hydrogen gas was also reported by another group ${ }^{21}$. In fact, for MWNT production, a gas that includes hydrogen atoms is more effective than an inert gas, such as $\mathrm{He}$ or Ar. The reason might be the high temperature and high activity of the hydrogen arc.

The MWNTs produced by hydrogen arc discharge $\left(\mathrm{H}_{2}\right.$-arc MWNTs) contain very few coexisting carbon nanoparticles ${ }^{22}$. These nanoparticles are easily removed by infrared irradiation or heating in air at $500^{\circ} \mathrm{C}$. High-resolution transmission electron microscopy observation of the purified $\mathrm{H}_{2}$-arc MWNTs reveals that their crystallinity is very high (having regular graphene sheets at an interlayer spacing of $3.4 \AA$ ) and an inner diameter typically as low as $7 \AA$, which is equal to the diameter of $\mathrm{C}_{60}$. A tube of $3 \AA$ diameter has been found exist inside $\mathrm{H}_{2}$-arc MWNTs ${ }^{23}$. Moreover, a linear carbon chain has also been observed $^{24}$ inserted into an MWNT with an innermost tube diameter of $7 \AA$ A Surprisingly, both the $3 \AA$ tube and the carbon chain exist in the same $\mathrm{H}_{2}$-arc MWNTs.

Corresponding to these thin innermost tubes in $\mathrm{H}_{2}$-arc MWNTs, radial breathing modes $(\mathrm{RBM})$ in Raman spectra are observed ${ }^{25,26}$. It is well known $^{27}$ that the wave number of RBM is inversely proportional to the tube diameter. From the Raman spectra, it is clear that $\mathrm{H}_{2}$-arc MWNTs have an innermost tube diameter that is smaller than independently produced SWNTs. In the high wave number region $\left(\sim 1580 \mathrm{~cm}^{-1}\right)$ of the Raman spectra, isolated $\mathrm{H}_{2}$-arc MWNTs exhibit splitting of the G-band ${ }^{28}$, which is usually only found in SWNTs. This is the characteristic of the quantum confinement effect ${ }^{28}$ because of the very thin diameter of CNTs.

The experimental history of SWNTs ${ }^{13,14}$ started two years after the discovery of MWNTs. SWNTs were also first produced by arc discharge using a graphite anode containing a metal catalyst ( $\mathrm{Fe}$ or $\mathrm{Co}$ ); however, SWNTs were obtained from the soot in the gas phase not from the cathode deposit. Of course, evaporated metal nanoparticles also coexist with the SWNTs, which have to be purified later. 
Mass production of SWNTs by arc discharge was achieved by Journet $e t$ a $l^{29}$ using a bimetallic Ni-Y catalyst in He ambient gas. The method was effectively modified ${ }^{30}$ by using two graphite electrodes inclined at an angle of $30^{\circ}$ instead of the conventional $180^{\circ}$ alignment. This is known as the arc-plasma-jet method ${ }^{30}$ and yields SWNTs at a rate of $w 1$ $\mathrm{g} / \mathrm{min}$. Another method ${ }^{31}$, using an $\mathrm{Fe}$ catalyst instead of $\mathrm{Ni}-\mathrm{Y}$ and an $\mathrm{H}_{2}$-Ar gas mixture in place of $\mathrm{He}$, produces a partly aligned macroscopic net of SWNTs as long as $30 \mathrm{~cm}$. Because the Fe nanoparticles attached to the SWNT net are covered with a very thin layer of amorphous carbon, the SWNTs are easily purified by heating in air at $420^{\circ} \mathrm{C}$ and then rinsing in mild $\mathrm{HCl}$.

In order to produce SWNTs, the use of metal catalysts is a necessary condition. The activities of various kinds of metal catalysts $\left(\mathrm{Ni}, \mathrm{Co}, \mathrm{Fe}, \mathrm{Pt}, \mathrm{Pd}\right.$ etc.) have been investigated ${ }^{32}$ and the maximum yield is achieved with Ni-Co. It was also observed that the addition of $\mathrm{S}$ increases the catalytic effect of other metal catalysts ${ }^{33,34}$. Double-walled nanotubes (DWNTs) produced by arc discharge have also been reported ${ }^{35,36,37}$ under conditions similar to those of large-diameter SWNT formation. It may be noted that SWNTs are not found in the cathode deposit like MWNTs but in the chamber soot. Therefore, an ac arc discharge, in which both the electrodes evaporate alternately, can produce a higher yield of SWNTs ${ }^{38}$.

Generally, it is hard to grow aligned CNTs (SWNTs, DWNTs, or MWNTs) by arc discharge, although partial alignment of SWNTs can be achieved by convection ${ }^{31}$ or directed arc plasma ${ }^{39}$. On the other hand, the growth temperature of the arc-discharge method is higher than that of other CNT production methods. As a result, the crystallinity and perfection of arc-produced CNTs are generally high, and the yield per unit time is also higher than other methods.

\section{Laser ablation}

The laser vaporization method, which had been originally used as a source of clusters ${ }^{40}$ and ultrafine particles ${ }^{41,42}$ was developed for fullerene and CNT production by Smalley's group $^{43,44}$. It is evident that vaporization of carbon is essential for the production of CNTs. Among various vaporization devices the laser is suitable for materials with high boiling temperature elements such as carbon because of its high energy density. Therefore, when applied to carbon, formation of fullerene was discovered by mass spectroscopy. But the yield was too low for structural identification at that time. To produce large quantities of fullerenes and other nanomaterials, Smalley's group further developed the laser ablation method also known as the laser-furnace method ${ }^{43}$ together with an annealing system in 1992. Fullerenes with a soccer ball structure are produced only at higher furnace temperatures, underlining the importance of annealing for nanostructures ${ }^{43}$. These discoveries were applied to produce $\mathrm{CNTs}^{44}$ in 1996, especially SWNTs.

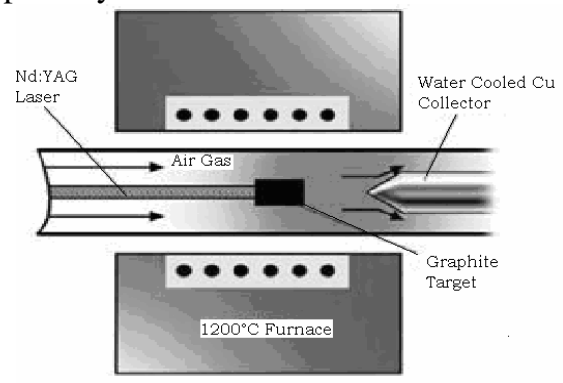

Figure 2. Schematic drawing of a laser ablation appratus 
The laser furnace (Figure 2), which consists of a furnace, a quartz tube with a window, a target carbon composite doped with catalytic metals, a water-cooled trap, and flow systems for the buffer gas to maintain constant pressures and flow rates ${ }^{43,44}$. A laser beam (typically a YAG or $\mathrm{CO}_{2}$ laser) is introduced through the window and focused onto the target located in the center of the furnace. The target is vaporized in high-temperature Ar buffer gas and forms SWNTs. The Ar flow rate and pressure are typically $1 \mathrm{~cm} \cdot \mathrm{s}^{-1}$ and 500 torr, respectively. The SWNTs produced are conveyed by the buffer gas to the trap, where they are collected. The vaporization surface is kept as fresh as possible by changing the focus point or moving the target.

The method has several advantages, such as high-quality SWNT production, diameter control, investigation of growth dynamics, and the production of new materials. High-quality SWNTs with minimal defects and contaminants, such as amorphous carbon and catalytic metals, have been produced using the laser-furnace method together with purification processes $45,46,47$. High crystallinity has been known to originate in high-power laser vaporization, homogeneous annealing conditions, and target materials without hydrogen ${ }^{32,48}$. The laser has sufficiently high energy density not to cleave the target into graphite particles but to vaporize it at the molecular level. The graphite vapor is converted into amorphous carbon as the starting material of SWNTs ${ }^{49,50,51}$. The annealing conditions of the amorphous carbon are more homogeneous than those of the arc-discharge method, in which the electrodes and the convection flow disturb the homogeneity of the temperature and flow rate ${ }^{51,52}$.

To achieve high quality DWNTs and SWNTs, a method called high-temperature pulsed arc discharge has been developed, which uses a dc pulsed arc discharge to maintain homogeneous condition in arc discharge inside a furnace ${ }^{53,54,55}$. It can be considered as hybrid of steady arc discharge and the laser-furnace method. The contaminants, such as amorphous carbon and catalytic metals present in CNTs were removed by purification processes based on oxidation by hot air $\left(400-500^{\circ} \mathrm{C}\right)$ or chemicals such as $\mathrm{H}_{2} \mathrm{O}_{2}{ }^{46}$. Amorphous carbon has many dangling bonds, which are not present in SWNTs, leading to high activity. High-quality SWNTs with few defects are more resistant to oxidation treatments than those of low quality. The oxidation treatments remove the protective layer of catalytic metal particles so that they are easily eliminated by acid treatments ${ }^{47}$.

SWNT diameter can be controlled by changing the furnace temperature, catalytic metals, and flow rate ${ }^{56}$. A higher furnace temperature results in SWNTs with larger diameters. The use of a Ni-Y alloy catalyst also increases the SWNT diameter, whereas a Rh-Pd catalyst reduces $i^{57}$. Flow rate affects the diameter distribution, which suggests that the growth process is fairly slow (on a timescale of seconds) compared with vaporization processes (nanosecond scale) ${ }^{50}$. SWNT growth is initiated by a short laser shot, so that the start time of the growth is defined. The growth processes have been traced by high-speed video imaging of light emission or scattering, from which the growth time is estimated to be more than several milliseconds ${ }^{51}$. A longer pulse duration, which leads to a lower energy density and lower vaporization temperature, is essential for the production process. The tunable duration of laser vaporization is another advantage in producing and exploring these nanoscale carbon materials. That is why the laser-furnace method is being used for growing various nanomaterials, and is expected to play a powerful role in nanotechnology. However, the laser technique is not economically advantageous because the process involves high purity graphite rods, high power lasers and low yield of CNTs.

\section{Chemical vapor deposition}

The chemical vapor deposition (CVD) is another popular method for producing CNTs in which a hydrocarbon vapor is thermally decomposed in the presence of a metal catalyst. The 
method is also known as thermal or catalytic CVD to distinguish it from the many other kinds of CVD used for various purposes. Compared with high temperature arc-discharge and laser methods, CVD is a simple and economic technique for synthesizing CNTs at relatively low temperature and ambient pressure, at the cost of crystallinity. It is versatile in that it harnesses a variety of hydrocarbons in any state (solid, liquid, or gas), enables the use of various substrates, and allows CNT growth in a variety of forms, such as powder, thin or thick films, aligned or entangled, straight or coiled, or even a desired architecture of nanotubes at predefined sites on a patterned substrate. It also offers better control over growth parameters.

In fact, CVD has been used for producing ${ }^{58,59,60}$ carbon filaments and fibers since 1959. Using the same technique, soon after the discovery of CNTs by Iijima, Endo et $\mathrm{al}^{61}$ reported CNT growth from pyrolysis of benzene at $1100^{\circ} \mathrm{C}$, while Jose-Yacaman et al. ${ }^{62}$ formed clear helical MWNTs at $700^{\circ} \mathrm{C}$ from acetylene. In both cases, Fe nanoparticles were used as the catalyst. Later, MWNTs were also grown from ethylene ${ }^{63}$, methane ${ }^{64}$ and many other hydrocarbons. SWNTs were first produced by Dai et al ${ }^{65}$ from disproportionation of CO at $1200^{\circ} \mathrm{C}$, catalyzed by Mo particles. Later, SWNTs were also produced from benzene ${ }^{66}$, acetylene $^{67}$, ethylene ${ }^{68}$, and methane ${ }^{69,70}$ using various catalysts.

Figure 3 shows a schematic diagram of the setup used for CNT growth by CVD in its simplest form. The process involves passing a hydrocarbon vapor (typically for 15-60 minutes) through a tube furnace in which a catalyst material is present at sufficiently high temperature $\left(600-1200^{\circ} \mathrm{C}\right)$ to decompose the hydrocarbon. CNTs grow over the catalyst and are collected upon cooling the system to room temperature. In the case of a liquid hydrocarbon (benzene, alcohol, etc.), the liquid is heated in a flask and an inert gas purged through it to carry the vapor into the reaction furnace. The vaporization of a solid hydrocarbon (camphor, naphthalene, etc.) can be conveniently achieved in another furnace at low temperature before the main, hightemperature reaction furnace. The catalyst material may also be solid, liquid, or gas and can be placed inside the furnace or fed in from outside. Pyrolysis of the catalyst vapor at a suitable temperature liberates metal nanoparticles in situ (the process is known as the floating catalyst method). Alternatively, catalyst-plated substrates can be placed in the hot zone of the furnace to catalyze CNT growth. Catalytically decomposed carbon species of the hydrocarbon are assumed to dissolve in the metal nanoparticles and, after reaching super saturation, precipitate out in the form of a fullerene dome extending into a carbon cylinder (like the inverted test tube shown in Figure 4) with no dangling bonds and, hence, minimum energy ${ }^{71,72}$. When the substrate-catalyst interaction is strong, a CNT grows up with the catalyst particle rooted at its base (known as the 'base growth model'). When the substrate-catalyst interaction is weak, the catalyst particle is lifted up by the growing CNT and continues to promote CNT growth at its tip (the 'tip growth model'). Formation of SWNTs or MWNTs is governed by the size of the catalyst particle. Broadly speaking, when the particle size is a few nanometers, SWNTs form, whereas particles a few tens of nanometers wide favor MWNT formation.

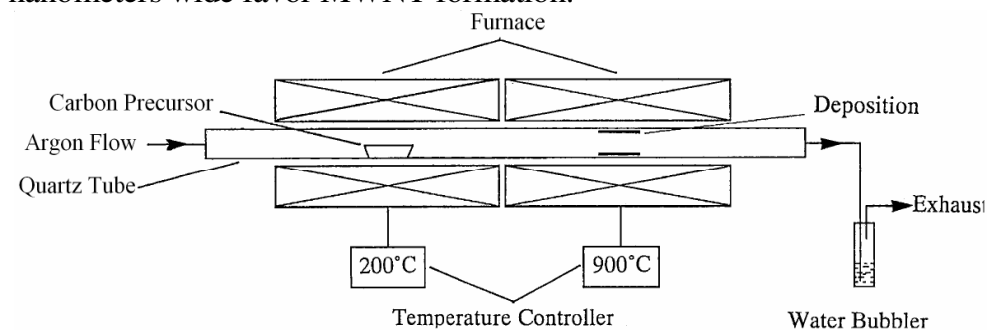

Figure.3. Schematic diagram of a CVD setup. 


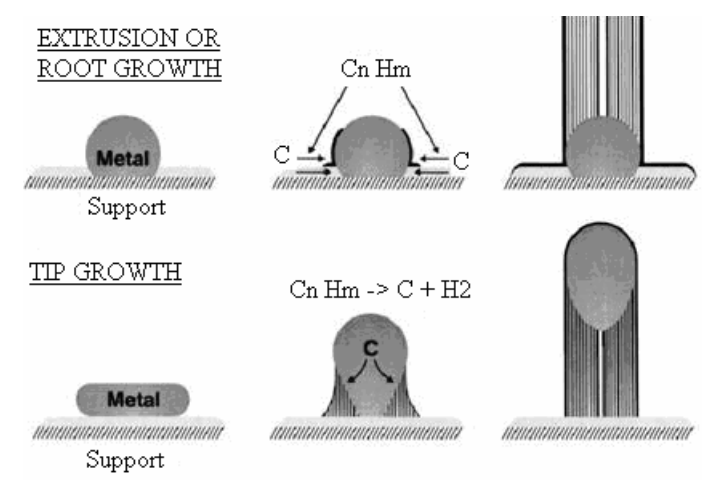

Figure 4. Probable models for CNT growth

The three main parameters for CNT growth in CVD are the hydrocarbon, catalyst, and growth temperature. General experience is that low-temperature CVD $\left(600-900^{\circ} \mathrm{C}\right)$ yields MWNTs, whereas a higher temperature $\left(900-1200^{\circ} \mathrm{C}\right)$ reaction favors SWNT growth, indicating that SWNTs have a higher energy of formation (presumably owing to their small diameters, which results in high curvature and high strain energy). This could explain why MWNTs are easier to grow from most hydrocarbons than SWNTs, which can only be grown from selected hydrocarbons (e.g. $\mathrm{CO}, \mathrm{CH}_{4}$, etc., that have a reasonable stability in the temperature range of $900-1200^{\circ} \mathrm{C}$ ). Common efficient precursors of MWNTs (e.g. acetylene, benzene, etc.) are unstable at higher temperatures and lead to the deposition of large amounts of carbonaceous compounds other than CNTs.

The growth of CNTs is catalyzed by transition metals. The metals such as $\mathrm{Fe}, \mathrm{Co}, \mathrm{Ni}$ are most commonly used for CNT growth, since the phase diagram of carbon and these metals suggests finite solubility of carbon in these transition metals at high temperatures. On saturation the carbon precipitates out. This leads to the formation of CNTs under the growth mechanism outlined above. It is remarkable that transition metals have proven to be efficient catalysts not only in CVD but also in arc-discharge and laser methods. This indicates that these apparently different methods might have a common growth mechanism for CNTs, which is not yet clear. The catalyst particle size has been found to dictate the tube diameter. Hence, metal nanoparticles of controlled size can be used to grow CNTs of controlled diameter ${ }^{73}$. Therefore, catalyst materials such as solid organometallocenes (ferrocene, cobaltocene, nickelocene) which liberate nanometal particles in-situ are used to catalyze CNT growth. Thin films of catalyst coated onto various substrates have also proved successful in achieving uniform CNT deposits ${ }^{74}$. In addition, the material, morphology, and textural properties of the substrate greatly affect the yield and quality of the resulting CNTs. Substrates such as silica, quartz, alumina and zeolite are mostly used. Zeolite support with catalysts in their nanopores has resulted in significantly higher yields of CNTs with a narrow diameter distribution ${ }^{75}$. Alumina materials are reported to be better catalyst supports than silica owing to their strong metal-support interaction, which allows high metal dispersion and thus, a high density of catalytic sites ${ }^{76}$. Such interactions prevent metal species from aggregating and forming unwanted large clusters that lead to graphite particles or defective MWNTs. The key to obtaining high yields of pure CNTs is achieving hydrocarbon decomposition on catalyst sites alone and avoiding spontaneous pyrolysis. CNTs have been successfully synthesized using organometallic compounds (nickel phthalocyanine ${ }^{77}$ and ferrocene ${ }^{78}$ ) as carbon-cum-catalyst precursors, though the as-grown CNTs were mostly metal encapsulated. The use of ethanol has drawn attention for synthesizing SWNTs at 
relatively low temperatures $\left(\sim 850^{\circ} \mathrm{C}\right)$ on $\mathrm{Fe}$-Co impregnated zeolite supports and Mo-Co coated quartz substrates ${ }^{79,80,81}$. Recently, a tree product, camphor, has been used to produce high yields of high purity MWNTs ${ }^{82,83,84}$. The MWNTs with least contamination of amorphous carbon and metal particles is achieved, as oxygen atom present in camphor help oxidize amorphous carbon in-situ and low catalyst requirement with camphor ${ }^{85}$. CVD is ideally suited to growing aligned CNTs on desired substrates for specific applications, which is not feasible by arc or laser methods. Li et $a l^{86}$ have grown dense MWNT arrays on Feimpregnated mesoporous silica prepared by a sol-gel process, terrones et $a l^{87}$ have produced CNTs on co-coated quartz substrates via CVD of a triazene compound with nearly no byproducts, while Pan et $\mathrm{ll}^{88}$ have reported the growth of aligned CNTs of more than $2 \mathrm{~mm}$ in length over mesoporous substrates from acetylene. Highly aligned nanotubes for electronics have been grown from acetylene ${ }^{89}$ using a Co catalyst impregnated in alumina nanochannels at $650^{\circ} \mathrm{C}$, while pillars of parallel CNTs have been grown from ethylene on Fe-patterned Si plates at $700^{\circ} \mathrm{C}$ for field emission applications. Bearing in mind that pyrolysis of a xylene-ferrocene mixture leads to the growth of vertical CNTs on quartz ${ }^{90}$, Ajayan and coworkers have produced organized assemblies of CNTs on thermally oxidized Si wafers ${ }^{91,92}$.

Since CVD is a well known and well established industrial process, CNT production is easy to scale up. MWNTs of controlled diameter are being produced in large quantities ( $\sim 100$ $\mathrm{g} /$ day) from acetylene using nanoporous materials as the catalyst support ${ }^{93}$. Wang et al $^{94}$ have developed a nano-agglomerate fluidized-bed reactor (a quartz cylinder $1 \mathrm{~m}$ long and $0.25 \mathrm{~m}$ wide) in which the continuous decomposition of ethylene gas on an Fe/alumina catalyst at $700^{\circ} \mathrm{C}$ produces a few kilograms of MWNTs per hour with a reported purity of $70 \%$. Dai's group has scaled up SWNT production from methane using a Fe-Mo bimetallic catalyst supported on a sol-gel derived alumina-silica multicomponent material ${ }^{95}$. However, Smalley's lab still leads the way in the mass production of SWNTs ( $\sim 10 \mathrm{~g} /$ day) by the high pressure carbon monoxide (HiPco) technique ${ }^{96}$. In this method, a Fe pentacarbonyl catalyst liberates Fe particles in situ at high temperatures, while a high pressure of $\mathrm{CO}$ ( $\sim 30 \mathrm{~atm})$ enhances the carbon feedstock manifolds, which significantly speeds up the disproportionation of $\mathrm{CO}$ molecules into carbon atoms and accelerates SWNT growth.

Apart from large-scale production, CVD also offers the possibility of growing single nanotubes for use as probe tips in atomic force microscopes (AFM) or as field emitters in electron microscopes. Hafner et $a l^{97}$ have grown single SWNTs and MWNTs (1-3 nm in diameter) rooted in the pores of Si tips suitable for AFM imaging. In another approach, single SWNTs are grown directly onto pyramids of Si cantilever-tip assemblies ${ }^{98}$. In this case, a SWNT grown on the Si surface (controlled by the catalyst density on the surface) protrudes from the apex of the pyramid. As-grown CNT tips are smaller than mechanically assembled nanotube tips by a factor of three and enable significantly improved resolution. CVD-produced CNTs have great promise for the fabrication of sophisticated instruments and nanodevices.

\section{Conclusions}

Carbon nanotubes clearly constitute a fascinating class of materials exhibiting a variety of novel properties. Synthesis is the key issue of CNT research. There has been wide variety of techniques for synthesizing CNTs. In this article, the different synthesis methods of CNTs have been reviewed. The CNTs (single and multiwalled) are produced by three main techniques, arc discharge, laser vaporization and chemical vapor deposition. The CNTs from the arc discharge are often covered with amorphous carbon, which contains metallic 
particles in the case of metal-carbon co-evaporation. The yield of CNTs from arc discharge is not very high, whereas with laser vaporization, the yield is much higher, but the quantities are small. With the laser vaporization method, CNTs are very clean i.e. less covered with amorphous carbon. The yield and shape of CNTs prepared by laser vaporization are determined by fewer parameters than are tubes obtained by arc discharge synthesis. Catalytic CVD is an extremely versatile technique for the production of CNTs. One major advantage of the CVD approach is that CNTs can be made continuously, which could provide a very good way to synthesize large quantities of CNTs under relatively controlled conditions. A variety of hydrocarbons, catalyst and catalyst supports have been used successfully by various groups worldwide to synthesize CNTs. Many investigations into the properties of CNTs and their potential applications require clean and uniform CNTs that contain no impurities. A number of purification methods have been developed to date. Care should be taken when the technique is chosen, as the effect on the entire sample will also depend on the composition and the amount of the sample. In last few years, significant progress has been made for fabricating various alignments and patterns of CNTs. The CVD technique can also be adopted for the controlled growth of CNTs at particular sites on a substrate for various applications. Due to the great potential of CNTs, it is clear that novel technologies will emerge in the near future.

\section{Reference}

1. Kroto H W, Heath J R, Obrin S C, Curl R F and Smalley R E, Nature, 1985, 318, 162.

2. Iijima S, Nature, 1991, 354, 56.

3. Ebbesen T W and Ajayan P M, Nature, 1992, 358, 220.

4. Kong J, Cassell A M and Dai H J, Chem Phys Lett., 1998, 292, 567.

5. Ebbesen T W, Hiura H, Fujita J, Ochiai Y, Matsui S and Tanigaki K, Chem Phys Lett., 1993, 209, 83.

6. Seraphin S, Zhou D, Jiao J, Withers J C and Loufty R, Carbon, 1993, 31, 685.

7. Michael Wilson, Kamali Kannangara, Geoff Smith and Michelle Simmons, Nanotechnology: Basic Science and Emerging Technologies; 2002.

8. Kratschmer W, Lowell D Lamp, Fostiropoulos K and Donald R Huffman, Nature, 1990, 347, 354

9. $\quad$ Ando Y and Iijima S, Jpn, J Appl Phys., 1993, 32, L107.

10. Yahachi Saito, Motonori Inagaki and Hisanori Shinohara, Chem Phys Lett., 1992, 200, 643.

11. Ebbesen T W, Carbon Nanotubes: Preparations and Properties; CRC Press, Baca Raton, FL, 1997.

12. Colbert D T, Zhang J, McClure S M, Nikolaev P, Chen Z, Hafner J H, Owens D W, Kotula P G, Carter C B, Weaver J H, Rinzler A G and Smalley R E, Science, 1994, 266 , 1218.

13. Iijima $\mathrm{S}$ and Ichihashi T, Nature, 1993, 363, 603.

14. Bethune D S, Kiang C H, De Vries M S, Gorman G, Savoy R, Vazquez J and Beyers R, Nature, 1993, 363, 605.

15. Ando Y, Jpn J Appl Phys., 1993, 32, L1342.

16. Ando Yoshinori, Fullerene Sci Tech., 1994, 2, 173.

17. Tai Y, Inukai K, Osaki T, Tazawa M, Murakami J, Tanemura S and Ando Y Chem Phys Lett., 1994, 224(1-2), 118.

18. Miao Wang, Xinluo Zhao, Masato Ohkohchi and Yoshinori Ando, Fullerene Sci, Tech., 1996, 4, 1027.

19. Ando Y, Zhao X and Ohkohchi M, Carbon, 1997, 35 (1), 153. 
20. Zhao X, Ohkohchi M, Wang M, Iijima S, Ichihashi T and Ando Y, Carbon, 1997, 35(6), 775.

21. Wang X K , Lin X W, Dravid V P, Ketterson J B, and Chang R P H, Appl Phys Lett.,1995, 66 (18), 2430.

22. Ando Y, Zhao X and Ohkohchi M, Jpn, J Appl Phys., 1998, 37, L61.

23. Zhao X, Liu Y, Inoue S, Suzuki T, Jones R O and Ando Y, Phys Rev Lett, 2004, 92, 125502.

24. Xinluo Zhao, Yoshinori Ando, Yi Liu, Makoto Jinno, and Tomoko Suzuki, Phys Rev Lett., 2003, 90, 187401.

25. Zhao X and Ando Y, Jpn, J Appl Phys., 1998, 37, 4846.

26. Xinluo Zhao, Yoshinori Ando, Lu-Chang Qin, Hiromichi Kataura, Yutaka Maniwa and Riichiro Saito, Chem Phys Lett., 2002, 361 (1-2), 169.

27. Bandow S, Asaka S, Saito Y, Rao A M, Grigorian L, Richter E and Eklund PC, Phys Rev Lett., 1998, 80 (17), 3779.

28. Zhao X, Ando Y, Qin L C, Kataura H, Maniwa Y and Saito R, Appl Phys Lett., 2002, 81(14), 2550.

29. Journet C, Maser W K, Bernier P, Loiseau A, Lamy de la Chapelle M, Lefrant S, Deniard P, Lee R and Fischer J E, Nature, 1997, 388, 756.

30. Yoshinori Ando, Xinluo Zhao, Kaori Hirahara, Kazutomo Suenaga, Shunji Bandow and Sumio Iijima, Chem Phys Lett., 2000, 323(5-6), 580.

31. Xinluo Zhao, Sakae Inoue, Makoto Jinno, Tomoko Suzuki and Yoshinori Ando, Chem Phys Lett., 2003, 373(3-4), 266.

32. Yudasaka M, Kasuya Y, Kokai F, Takahashi K, Takizawa M, Bandow S and Iijima S, Appl Phys A., 2002, 74 (3), 377.

33. Liu C, Cong H T, Li F, Tan P H, Cheng H M, Lu K and Zhou B L, Carbon 1999, 37 (11), 1865.

34. Ando Y et al., Nanonetwork Materials, AIP CP590 2001,11, 7.

35. Hutchison J L, Kiselev N A, Krinichnaya E P, Krestinin A V, Loutfy R O, Morawsky A P, Muradyan V E, Obraztsova E D, Sloan J, Terekhov S V and Zakharov D N, Carbon, 2001, 39(5), 761.

36. Saito Y, Nakahira T and Uemura S, J Phys Chem., B 2003, 107(4), 931.

37. Sugai T, Yoshida H, Shimada T, Okazaki T, Shinohara H and Bandow S, Nano Lett., 2003, 3(6), 769.

38. Ohkohchi M, Jpn J Appl Phys., 1999, 38, 4158.

39. Houjin Huang, Hisashi Kajiura, Shigemitsu Tsutsui, Yoshiyuki Hirano, Mitsuaki Miyakoshi, Atsuo Yamada and Masafumi Ata, Chem Phys Lett., 2001, 343(1-2), 7.

40. Powers D E, Hansen S G, Geusic M E, Puiu A C, Hopkins J B, Dietz T G, Duncan M A, Langridge-Smith P R R and Smalley R E, J Chem Phys., 1982, 86, 2556.

41. In: Bernstein E R, Editors, Atomic and Molecular Clusters, Elsevier Science B V; New York, 1990, 69.

42 Uyeda R, Prog Mater Sci., 1991, 35(1), 1.

43. Ting Guo, Diener M D, Yan Chai, Alford M J, Haufler R E, McClure S M, Ohno T, Weaver J H, Scuseria G E, and Smalley R E, Science, 1992, 257, 1661.

44. Thess A, Lee R, Nikolaev P, Dai H, Petit P, Robert J, Xu C, Lee Y H, Kim S G, Rinzler A G, Colbert D T, Scuseria G E, Tomalnek D, Fischer J E and Smalley R E, Science, 1996, 273, 483.

45. Bandow, Shunji, Rao A M, Williams K A, Thess A, Smalley R E and Eklund P C, $J$ Phys Chem., 1997, 101(44), 8839. 
46. Chiang I W, Brinson B E, Huang A Y, Willis P A, Bronikowski M J, Margrave J L, Smalley R E and Hauge R H, J Phys Chem., B 2001, 105(35), 8297.

47. Ishii H, Kataura H, Shiozawa H, Yoshioka H, Otsubo H, Takayawa Y, Miyahara T, Suzuki S, Achiba Y, Nakatake M, Narimura T, Higashiguchi M, Shimada K, Namatame H and Taniguchi M, Nature, 2003, 426, 540.

48. Shinohara H, Rep Prog Phys., 2000, 63(6), 843.

49. Puretzky A A, Geohegan D B, Fan X and Pennycook S J, Appl Phys., A 2000, 70(2), 153.

50. Rahul Sen Y, Ohtsuka T, Ishigaki D, Kasuya, S Suzuki H, Kataura Y and Achiba, Chem Phys Lett., 2000, 332 (5-6), 467.

51. Kokai F, Takahashi K, Yudasaka M and lijima S, J Phys Chem., B 2000, 104(29), 6777.

52. Kanai M, Koshio A, Shinohara H, Mieno T, Kasuya A, Ando Y and Zhao X, Appl Phys Lett., 2001, 79(18), 2967.

53. Sugai, Toshiki, Omote, Hideki, Bandow, Shunji, Tanaka, Nobuo, Shinohara and Hisanori, Jpn J Appl Phys., 1999, 38, L477.

54. Sugai T, Omote H, Bandow S, Tanaka N and Shinohara H, J Chem Phys., 2000, 112 (13), 6000 .

55. Shimada T, Sugai T, Ohno Y, Kishimoto S, Mizutani T, Yoshida H, Okazaki T and Shinohara H, Appl Phys Lett., 2004, 84 (13), 2412.

56. Kataura H, Kumazawa Y, Maniwa Y, Ohtsuka Y, Sen R, Suzuki S and Achiba Y, Carbon, 2000, 38(11-12), 1691.

57. Kataura H, Kimura A, Ohtsuka Y, Suzuki Sh, Maniwa Y, Hanyu T and Achiba Y, Jpn J Appl Phys., 1998, 37, L616

58. Walker Jr P L, Rakszawski J F and Imperial G R, J Phys Chem., 1959, 63(2), 133.

59. In: Dresselhaus M S, Editors, Graphite fibers and filaments, Springer; Berlin, 1988.

60. Endo M, Chemtech., 1988, 18, 568.

61. Morinobu Endo, Kenji Takeuchi, Susumu Igarashi, Kiyoharu Kobori, Minoru Shiraishi and Harold W Kroto, J Phys Chem Solids., 1993, 54(12), 1841.

62. Jose Me Yacaman, Miki M Yoshida and L. Rendon L, Appl Phys Lett., 1993, 62(6), 657.

63. Satishkumar B C, Govindaraj A and Rao C N R, Chem Phys Lett., 1999, 307(2), 158.

64. Hernadi K, Fonseca A, Nagy J B, Bernaerts D and Lucas A A, Carbon, 1996, 34(10), 1249.

65. Hongjie Dai, Andrew G. Rinzler, Pasha Nikolaev, Andreas Thess, Daniel T Colbert and Richard E Smalley, Chem Phys Lett., 1996, 260(3-4), 471.

66. Cheng H M, Li F, Sun X, Brown S D M, Pimenta M A, Marucci A, Dresselhaus G and Dresselhaus M S, Chem Phys Lett., 1998, 289(5-6), 602.

67. Satishkumar B C, Govindaraj A, Rahul Sen and Rao C N R, Chem Phys Lett., 1998, 293(1-2), 47.

68. Jason H Hafner, Michael J Bronikowski, Bobak R Azamian, Pavel Nikolaev, Andrew G Rinzler, Daniel T Colbert, Ken A Smith and Richard E Smalley, Chem Phys Lett., 1998, 296 (1-2), 195.

69. Jing Kong, Alan M. Cassell and Hongjie Dai, Chem Phys Lett., 1998, 292(4-6), 567.

70. Flahaut E, Govindaraj A, Peigney A, Laurent Ch, Rousset A and Rao C N R, Chem Phys Lett., 1999, 300(1-2), 236.

71. Baker R T K In: P L Walker and P A Thrower, Editors, Chemistry and Physics of Carbon, Marcel Dekker; New York 1978, 14.

72. Gary G Tibbetts, J Cryst Growth, 1984, 66(3), 632.

73. Hiroki Ago, Toshiki Komatsu, Satoshi Ohshima, Yasunori Kuriki and Motoo Yumura, Appl Phys Lett., 2000, 77, 79. 
74. Shoushan Fan, Michael G Chapline, Nathan R Franklin, Thomas W Tombler, Alan M Cassell and Hongjie Dai, Science., 1999, 283, 512.

74. Hernadia K, Fonsecaa A, Nagya J B, Bernaertsc D, Fudalab A and Lucas A A, Zeolites., 1996, 17(5-6), 416.

76. Nagaraju N, Fonseca A, Konya Z and Nagy J B, J Mol Catal., A, 2002, 181(1-2), 57.

77. Yudasaka, Kikuchi R, Ohki Y and Yoshimura S, Carbon., 1997, 35 (2), 195.

78. Rao C N R, Sen R, Satishkumar B C and Govindaraj A, Chem Commun., 1998, 15, 1525.

79. Shigeo Maruyama, Ryosuke Kojima, Yuhei Miyauchi, Shohei Chiashi and Masamichi Kohno, Chem Phys Lett., 2002, 360(3-4), 229.

80. Yoichi Murakami, Yuhei Miyauchi, Shohei Chiashi and Shigeo Maruyama, Chem Phys Lett., 2003, 377(1-2), 49.

81. Yoichi Murakami, Shohei Chiashi, Yuhei Miyauchi, Minghui Hu, Masaru Ogura, Tatsuya Okubo and Shigeo Maruyama, Chem Phys Lett., 2004, 385(3-4), 298.

82. Mukul Kumar and Yoshinori Ando, Diamond Relat Mater., 2003, 12(3-7), 998.

83. Mukul Kumar and Yoshinori Ando, Chem Phys Lett., 2003, 374(5-6), 521.

84. Mukul Kumar, Keita Kakamu, Tsugio Okazaki and Yoshinori Ando, Chem Phys Lett., 2004, 385(3-4), 161.

85. Mukul Kumar and Yoshinori Ando, Diamond Relat Mater, 2003, 12(10-11), 1845.

86. Li W Z, Xie S S, Qian L X, Chang B H, Zou B S, Zhou W Y, Zhao R A and Wang G, Science., 1996, 274, 1701.

87. Terrones M, Grobert N, Olivares J, Zhang J P, Terrones H, Kordatos K, Hsu W K, Hare J P, Townsend P D, Prassides K, Cheetham A K, Kroto H W and Walton D R M, Nature, 1997, 388, 52.

88. Pan Z W, Xie S S, Chang B H, Wang, C Y, Lu L, Liu W, Zhou W Y, Li W Z and Qian L X, Nature, 1998, 394, 631.

89. Li J, Papadopoulos C, Xu J M and Moskovits M, Appl Phys Lett., 1999, 75(3), 367.

90. Andrews R, Jacques D, Rao A M, Derbyshire F, Qian D, Fan X, Dickey E C and Chen J, Chem Phys Lett., 1999, 303(5-6), 467.

91. Wei B Q, Vajtai R, Jung Y, Ward J, Zhang R, Ramanath G and Ajayan P M, Nature, 2002, 416, 495.

92. Cao A, Ajayan P M, Ramanath G, Baskaran R and Turner K, Appl Phys Lett., 2004, 84 (1), 109.

93. Couteau E, Hernadi K, Seo J W, Thien-Nga T, Miko Cs, Gaal R and Forro L, Chem Phys Lett., 2003, 378(1-2), 9.

94. Yong Wang, Kouhei Tanaka, Toshiyuki Nakaoka and Kazuo Murase, Chem Phys Lett., 2002, 364(5-6), 568.

95. Alan M cassel, Jeffrey A raymakess, Jing kong and hongjie dai, J Phys Chem., B, 1999, 103(31), 6484.

96. Michael J Bronikowski, Peter A Willis, Daniel T Colbert, Smith K A and Richard E Smalley, J Vac Sci Technol., A, 2001, 19(4), 1800.

97. Hafner J H, Cheung C L and Lieber C M, Nature, 1999, 398, 761.

98. Jason H Hafner, Chin Li Cheung and Charles M Lieber, J Am Chem Soc., 1999, 121(41), 9750. 


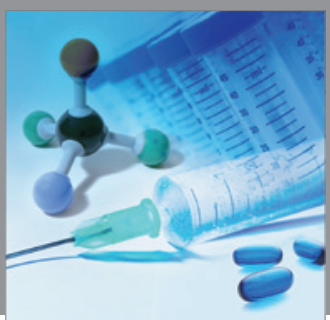

International Journal of

Medicinal Chemistry

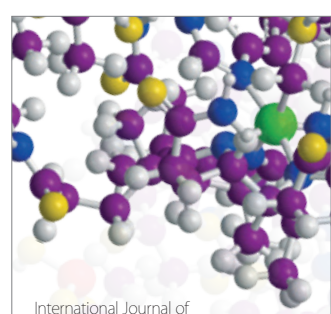

Carbohydrate Chemistry

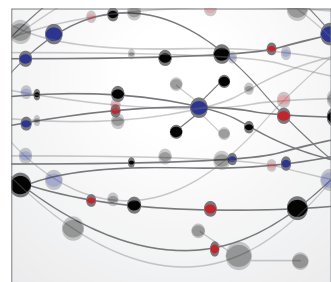

The Scientific World Journal
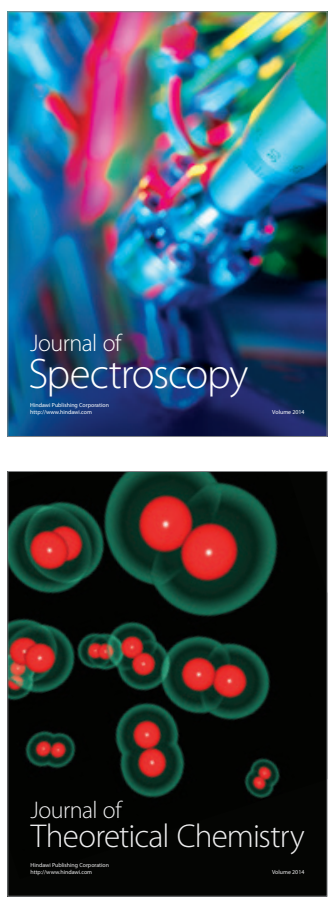
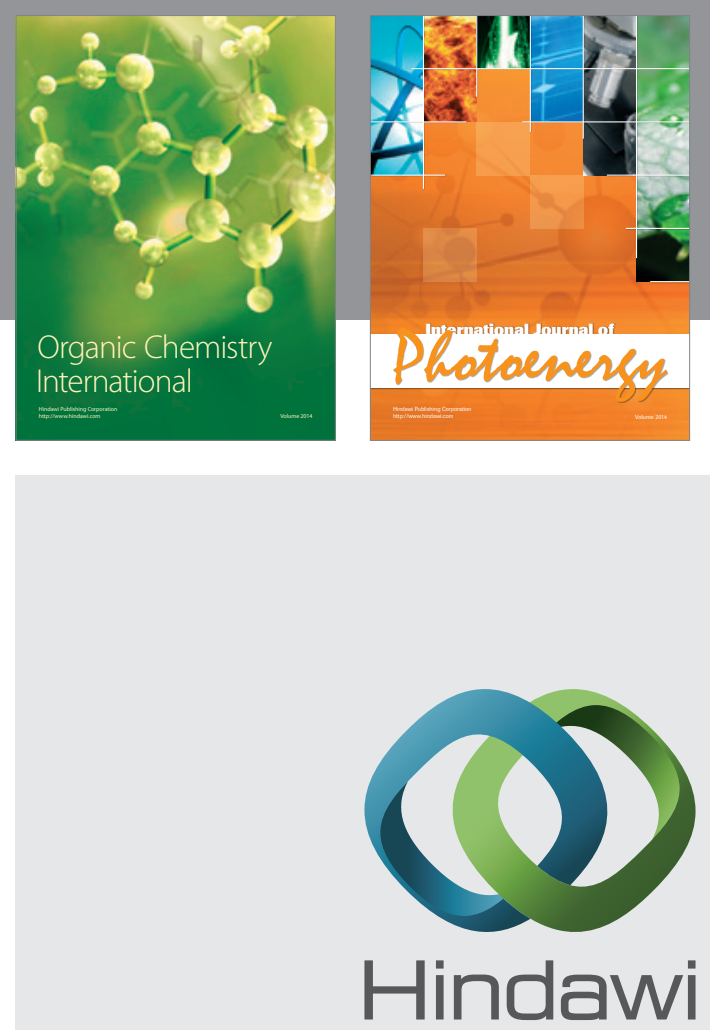

Submit your manuscripts at

http://www.hindawi.com
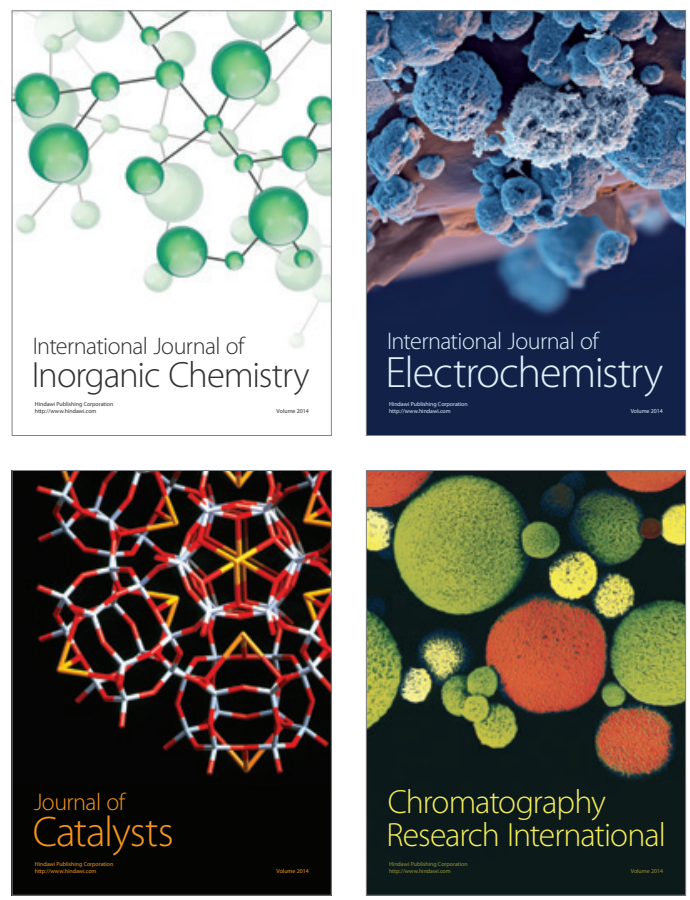
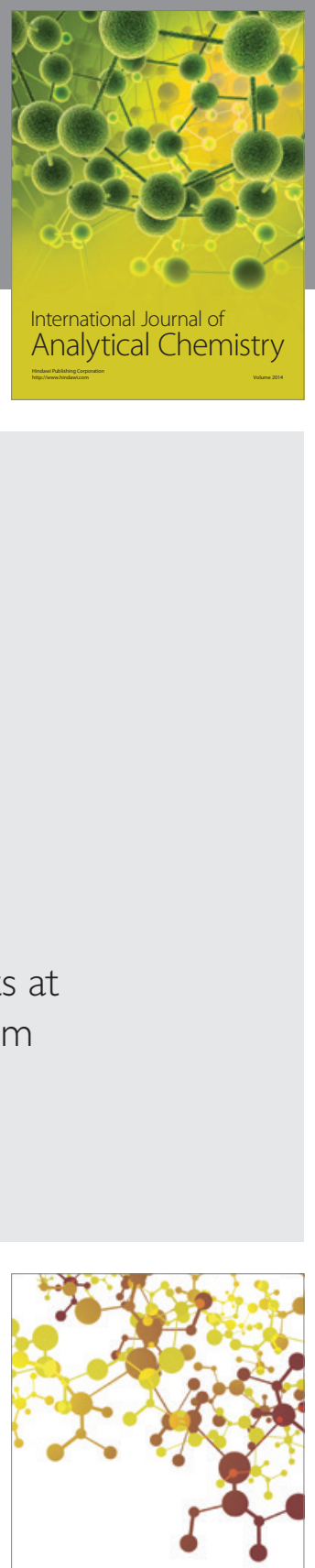

Journal of

Applied Chemistry
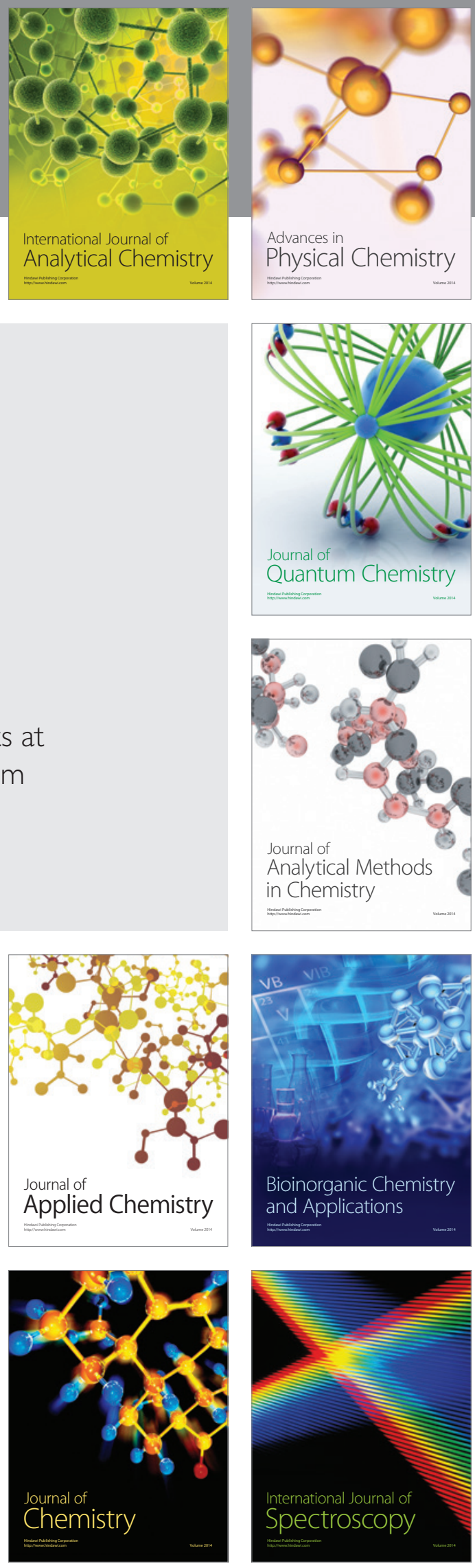